\title{
PERCEPCIÓN E INTERESES DEL ADOLESCENTE ANTE DISTINTAS INTERROGANTES Y TOMA DE DECISIONES COMO ESTUDIANTE DE SECUNDARIA
}

\author{
PERCEPTION AND INTERESTS OF THE ADOLESCENT TO \\ DIFFERENT QUESTIONS AND DECISION MAKING AS A SECONDARY \\ STUDENT
}

Esteban Pérez López ${ }^{1}$

\begin{tabular}{|l|l} 
Recibido: 17.05 .18 & Aprobado: 02.11 .18
\end{tabular}

DOI: $10.15517 /$ isucr.v20i42.41847

\begin{abstract}
Resumen
Los estudiantes de secundaria enfrentan un cúmulo de experiencias y decisiones determinantes a una edad temprana en medio de un entorno exigente y contemporáneo. Se indagó con respecto a su afinidad y dificultad por las principales materias, la modalidad preferida para generar su conocimiento, su afinidad por el uso de dispositivos electrónicos, redes sociales e internet, su proyección para la decisión de cursar estudios superiores, y otros. El estudio se realizó en tres diferentes colegios de la provincia de Alajuela (Grecia, Atenas, Pacto del Jocote) en Costa Rica.

Para la captura de la información se diseñó un instrumento de consulta (cuestionario), el cual se aplicó de manera física (en papel) a estudiantes de todos los niveles de secundaria.

Los resultados obtenidos destacan que el $64 \%$ de los adolescentes incluidos en el estudio consideran la Matemática como la materia más difícil. Un 47\% de los adolescentes eligieron el área de las Ciencias como la materia que más les gusta. El 58\% de los adolescentes prefieren la asignación de trabajos en grupo y exposición de los estudiantes como la opción más adecuada para el proceso enseñanza-aprendizaje en el aula. También destaca que todos los encuestados tienen teléfono celular, el 95\% tienen internet en su celular, un 94\% de ellos usan Facebook y 54\% usan Whatsapp. El 81\% de los estudiantes ya tiene claro que les gustaría estudiar en educación superior. El principal motivo de elección de carrera sería por la afinidad con la profesión con un $30 \%$, seguido del buen salario que ostenta el profesional con un $26 \%$.
\end{abstract}

\footnotetext{
1 Costarricense. Docente e investigador. Sede Occidente. Recinto de Grecia. Máster en Sistemas Modernos de Manufactura y Bach. en Laboratorista Químico. Profesor UCR-Recinto Grecia. esteban.perezlopez@ucr.ac.cr
} 
Palabras Clave: Educación; adolescentes; estudiantes; secundaria; decisiones y realidades.

\begin{abstract}
High school students face a wealth of experiences and decisive decisions at an early age amid a demanding and contemporary environment. It was investigated regarding their affinity and difficulty for the main subjects, the preferred modality to generate their knowledge, their affinity for the use of electronic devices, social networks and the Internet, their projection for the decision to pursue higher education, and others. The study was carried out in three different schools in the province of Alajuela (Grecia, Atenas, Pacto del Jocote) in Costa Rica.

To capture the information, a consultation instrument (questionnaire) was designed, which was applied physically (on paper) to students at all levels of secondary school.

The results obtained highlight that $64 \%$ of the adolescents included in the study consider Mathematics as the most difficult subject. $47 \%$ of adolescents chose the Science area as the subject they like the most. 58\% of adolescents prefer assigning group work and exposing students as the most appropriate option for the teaching-learning process in the classroom. It also highlights that all respondents have a cell phone, $95 \%$ have internet on their cell phone, $94 \%$ of them use Facebook and 54\% use WhatsApp. $81 \%$ of students are already clear that they would like to study in higher education. The main reason for choosing a career would be due to the affinity with the profession with $30 \%$, followed by the good salary of the professional with $26 \%$.
\end{abstract}

Keywords: Education; adolescents; students; secondary; decisions and realities.

\title{
Introducción
}

Los adolescentes son una fuente de creatividad, de iniciativa, de dinamismo y de renovación social. Si cuentan con la educación adecuada, podrán contribuir al desarrollo económico y al progreso de la sociedad. Por el contrario, si los adolescentes sienten que la enseñanza que reciben es distante y sin sentido, si conciben a la escuela o el liceo sólo como un espacio social de encuentro con los amigos y si su principal queja es que no son escuchados por los maestros y los adultos en general, entonces hay que plantearse una reforma radical y creativa de los centros educativos (Artagaveytia \& Bonetti, 2006).

En los lugares donde la educación secundaria está disponible, las comunidades se benefician de una mayor participación ciudadana, de niveles más bajos en violencia juvenil, de la reducción en la pobreza y de un mayor empoderamiento social (Unicef, 2011).

Es fundamental invertir en la educación y capacitación ya que se conoce que la educación secundaria aumenta los ingresos individuales potenciales y el crecimiento económico general. En un mercado laboral cada vez más tecnológico, la educación superior proporciona las capacidades necesarias para aumentar la productividad y estimular la inversión extranjera. La educación secundaria también tiene un fuerte impacto en la promoción de la igualdad de género y el 
mejoramiento de la salud materna. Las adolescentes que asisten a la escuela secundaria tienen seis veces menos probabilidades de casarse en esa edad y tres veces menos probabilidad de quedar embarazadas (Unicef, 2011).

Frente a los numerosos desafíos del porvenir, la educación constituye un instrumento indispensable para que la humanidad pueda progresar hacia los ideales de paz, libertad y justicia social (Delors, Amagi, Carneiro, Chung, Geremek, Gorham, \& Stavenhagen, 1997).

La educación es un bien público que hace posibles las transformaciones. Es una herramienta de equidad y una de las estrategias más potentes para alcanzar el desarrollo y consolidar sociedades pacíficas, solidarias y democráticas. No obstante, hablamos de educación de calidad como un derecho fundamental ineludible, pero lejano todavía del alcance de todos (Artagaveytia \& Bonetti, 2006).

Unicef (2011) relata que la educación secundaria es fundamental para el desarrollo y el bienestar del adolescente. Para superar con éxito los múltiples riesgos que ponen en peligro su desarrollo y sus derechos, es necesario dotar a los adolescentes de una amplia gama de conocimientos y capacidades esenciales, entre ellos resolver problemas de una manera creativa, buscar y evaluar la información con espíritu crítico y comunicarse con eficacia.

Es en la práctica particular y en el vínculo entre el adulto y el adolescente que se generará un proceso de aprendizaje estimulante y motivador. Sólo así se legitimará ese proceso de transformación esperado (Artagaveytia \& Bonetti, 2006).

\section{Fundamento}

Artagaveytia \& Bonetti (2006) indican que la Convención sobre Derechos del Niño (CDN) define las condiciones mínimas que los Estados deben asegurar en cuanto al acceso a los distintos ciclos educativos: enseñanza primaria obligatoria y gratuita para todos, enseñanza secundaria y orientación profesional con diversidad de formas disponibles y accesibles para todos y enseñanza superior accesible a todos.

La educación secundaria ha tenido en la mayoría de países del mundo un papel estratégico en la dinámica, composición y funcionamiento de los sistemas educativos; en garantizar la educación obligatoria a las distintas poblaciones nacionales y ha sido también un espacio de formación fundamental, tanto para preparar a los adolescentes para la vida universitaria, como para 
proveer de algunas competencias fundamentales de orden técnico y profesional para vincular a los jóvenes en la vida laboral (López \& Angulo, 2006).

Krichesky (2005), menciona que en la región de América Latina se observan logros educativos en términos de expansión y cobertura, que aunque han sido muy importantes, siguen siendo insuficientes. Estos avances se combinan con una persistente segmentación que afecta a los más pobres y a los que habitan en zonas rurales, lo que evidencia políticas de democratización educativa deficitarias. En el año 2002, apenas el 34.8\% de los jóvenes de 20 a 24 años habían completado la secundaria, mientras que en el segmento de 15 a 29 años de edad el egreso de primaria llegó a $66.7 \%$. Por lo que persisten las disparidades entre jóvenes por grupos de ingresos y localización geográfica.

Para Delors et al (1997), la educación básica llega en todo el mundo a los 900 millones de adultos analfabetos, a los 130 millones de niños sin escolarizar y a los más de 100 millones de niños que abandonan la escuela antes de tiempo. A ellos deben dirigirse de forma prioritaria las actividades de asistencia técnica y de coparticipación en el marco de la cooperación internacional.

Alcázar, Rendón \& Wachtenheim (2001), encuentran que el ingreso y nivel educativo de los padres, así como la composición del hogar son importantes determinantes de las probabilidades de asistencia escolar y trabajo en las áreas rurales. Ello implica que mejores condiciones de vida en las áreas rurales de América Latina pueden tener un impacto considerable en aumentar el éxito educativo de sus habitantes y sus descendientes. Los adolescentes rurales se encuentran en extrema desventaja frente a sus pares urbanos. En general, pertenecen a hogares más pobres, sus padres son menos educados, sus familias son más numerosas, y habitan donde existe una relativa escasez de escuelas, profesores y materiales.

La educación se construye fundamentalmente a través de la interacción entre el sujeto social, la familia, los grupos de pares, la escuela, la sociedad y los medios de comunicación. Es decir, la educación en general y la educación de los adolescentes en particular, se ve construida e influida decisivamente por la socialización primaria y la socialización secundaria (Bertolino, 2011).

La educación básica es un problema que se plantea, lógicamente, en todos los países, incluidos los industrializados. Desde este nivel de la educación, los contenidos tienen que fomentar el deseo de aprender, el ansia y la alegría de conocer y, por lo tanto, el afán y las posibilidades de acceder más tarde a la educación durante toda la vida (Delors et al, 1997). 
Repensar la educación para los adolescentes en camino de mejorarla conduce, por un lado, a retomar cierto sentido común que focalice prioridades y, por otro lado, obliga a un máximo de creatividad e innovación para poder brindar nuevas alternativas (Krichesky, 2005).

Adicionalmente, sobre el papel de la escuela en la educación en el tiempo libre en adolescentes, un estudio reciente realizado con docentes españoles de secundaria; Fraguela, Varela \& Caballo (2016), indican que, casi la mitad del profesorado está bastante o muy de acuerdo con que los centros abran sus instalaciones fuera del horario lectivo de lunes a viernes, y un tercio de ellos opina favorablemente de que también se haga los fines de semana.

Habilidades, necesidades y decisiones en adolescentes

Muy ligado con la educación en los adolescentes, el desarrollo de habilidades les permite un crecimiento integral que maximiza su potencial en las diferentes actividades de la vida diaria.

En relación a la habilidad de toma de decisiones en adolescentes se menciona que esta habilidad es un proceso complicado, puesto que requiere de un análisis afectivo, emocional y cognitivo. Asimismo para la toma de decisiones, especialmente bajo condiciones de estrés, involucran habilidades de pensamiento cognitivo (identificación de asuntos o problemas, determinación de metas, generación de soluciones alternativas, imaginación de posibles consecuencias) y habilidades para enfrentar emociones (calmarse a sí mismo en situaciones de estrés, escuchar con exactitud y determinar la mejor opción). Para desarrollar la habilidad de toma de decisiones se requiere pensamiento crítico para evaluar y detectar un problema o una situación que requiere tomar una decisión. También se requiere pensamiento creativo para analizarlo y sintetizarlo, además de la capacidad de resolver un problema para encontrar alternativas de solución (Choque-Larrauri \& Chirinos-Cáceres, 2009).

Según estudio de Gómez-Vela, Verdugo \& González (2007), cuyos objetivos fueron: evaluar la calidad de vida y el autoconcepto, y comprobar la relación que existe entre estas dos variables durante la adolescencia. Estudio en el que participaron 1121 alumnos de entre 12 y 18 años; 151 presentaban necesidades educativas especiales (NEE). Los adolescentes presentan puntuaciones positivas tanto en calidad de vida como en autoconcepto. No obstante, existen diferencias significativas en función del género, la edad y la presencia de NEE. Los varones obtienen puntuaciones inferiores en el área académica, mientras que las mujeres las obtienen en la esfera emocional. Los alumnos más jóvenes obtienen puntuaciones inferiores en 
Autodeterminación. Las NEE afectan al autoconcepto académico y al desarrollo personal de los alumnos, a su bienestar físico y a su autodeterminación; el autoconcepto desempeña un papel importante en la calidad de vida durante la adolescencia, especialmente el autoconcepto familiar, el académico y el físico.

En cuanto a los adolescentes como estudiantes, Pacheco (2001) menciona algunas necesidades básicas del estudiante, que se mencionan a continuación:

$\checkmark$ Máximo logro de destrezas y conceptos básicos.

$\checkmark$ Actividades de aprendizaje a nivel y ritmo apropiado.

$\checkmark$ Experiencias en pensamiento creativo y solución de problemas.

$\checkmark$ Desarrollo de la capacidad convergente, especialmente de la deducción lógica y la solución de problemas.

$\checkmark$ Estimulación de la imaginación y las capacidades espaciales.

$\checkmark$ Desarrollo de la autoconciencia y aceptación de las capacidades, intereses y necesidades propias.

$\checkmark$ Estimulación para perseguir metas y aspiraciones de alto nivel.

$\checkmark$ Desarrollo de la independencia, autodirección y disciplina en el aprendizaje.

$\checkmark$ Experiencias de relación con otros estudiantes intelectual, artística y efectivamente muy capacitados, creativos y talentosos.

$\checkmark$ Amplias fuentes de información sobre diversos temas.

$\checkmark$ Exposición a una variedad de campos de estudio, arte, profesiones y ocupación.

$\checkmark$ Acceso y estimulación para la lectura.

Al analizar el concepto de la naturaleza del talento sobresaliente en el individuo, se determina que el desarrollo de las capacidades potenciales (cognitivas y especiales) es un proceso complejo, dinámico, que implica una interrelación de factores personales, familiares, sociales y culturales en el que desarrollan y se proyectan los atributos espirituales, intelectuales, emocionales y sociales de la persona que los posee (Pacheco, 2001).

En Costa Rica, el Código de la Niñez y la Adolescencia ha impactado y generado nuevos retos a las instituciones que tienen a su cargo la atención de este colectivo como población meta, sin embargo, el avance es desigual. Por ejemplo, el Ministerio de Salud ha sobresalido como institución pionera en dicha materia, pero existen problemas que se intentan solventar con recursos 
limitados. Por su parte, el Ministerio de Educación Pública, a pesar de trabajar sobre la mejora general del sistema educativo, ha tenido un aporte tangencial en temas referidos al abordaje de un auténtico enfoque de derechos, siendo que los primeros pasos que se están dando en esta dirección son muy recientes (Gutiérrez \& Piedra, 2008).

El estudio de García (2008), evidencia que la clase social parece relacionarse con la construcción y formación de la identidad personal de adolescentes escolarizados, mujeres y varones de 16-17 años en San José, Costa Rica. De forma tal que en la dimensión interpersonal los adolescentes de clase asalariada promedian más alto en la posición de realización, y los jóvenes de clase burguesa promedian más alto en forclusión. Se hipotetiza que esta diferencia puede relacionarse con el hecho de que los jóvenes de la clase asalariada están expuestos a las exigencias de su estado social, de resolver más tareas del desarrollo por sí mismos. Mientras que los jóvenes burgueses participan dentro de un espacio social que procura el mantenimiento y reproducción de su lugar hegemónico, y les brinda más recursos materiales, pero menos oportunidades para cuestionarse a sí mismos y al lugar que ocupan dentro de la sociedad.

Por su parte, el estudio de Vázquez \& Manassero (2008), presenta un análisis empírico de la hipótesis del deterioro de las actitudes relacionadas con la ciencia a medida que crece la edad de los estudiantes. Los factores actitudinales estudiados comprenden un conjunto de variables sobre imagen de la ciencia, preservación del medio ambiente y aspectos de la ciencia escolar, que se analizaron en una muestra de estudiantes de diversas edades desde el cuarto curso de primaria en adelante. La evolución temporal de las respuestas de los estudiantes demuestra el descenso global de las actitudes con la edad y el sexo: en los primeros años son más positivas y van disminuyendo al aumentar la edad y los chicos tienen mejores actitudes que las chicas. Principalmente, este descenso afecta a las actitudes hacia algunos aspectos de la ciencia escolar, mientras los aspectos generales de la imagen de la ciencia y la tecnología o la preservación del medio ambiente no exhiben este deterioro.

En cuanto al hábito lector y la afición por la lectura en adolescentes, Villaseñor (2006) indica que no dependen estrictamente de la presencia omnímoda de un entorno urbano o de la disponibilidad inmediata de unos recursos culturales imprescindibles y próximos al potencial joven lector, sino también de otros factores quizá de mayor consistencia, como son: la comunidad educativa, la labor de animación a la lectura que los profesores practiquen con sus alumnos desde 
edades tempranas, la predisposición ambiental, familiar y social del joven escolar y el óptimo aprovechamiento de su ocio, entre otros.

Aguilar, Lozoya \& Guzmán (2015), revelan en su estudio como dentro de la materia de Formación Cívica y Ética en ocasiones es difícil saber el impacto que tienen las actividades en el proceso de enseñanza aprendizaje de los adolescentes pero, se ha podido observar, con el trabajo por proyectos, que los alumnos logran ser sensibles ante ciertas situaciones de índole social, con base a la experiencia, contacto y acercamiento a estas situaciones. A través de la indagación el estudiante puede hacer propuestas y reflexionar sobre diversos saberes significativos. Porque precisamente el que elaboren cosas que a ellos les interese o incluso con la asignación de los temas, el simple hecho de construir en pares o en colectivo les permite profundizar, cuestionar o proponer, entre otras habilidades. Por ejemplo en el intercambio virtual, los alumnos pudieron darse cuenta, lo cual es un logro con el individualismo en las sociedades actuales, que la adolescencia no es universal y que cada uno la vive, la siente y la construye de forma diferente.

En cuanto a la enseñanza de las ciencias, Quintanal-Pérez (2012) asevera que es fundamental ensamblar los estilos de enseñanza de los profesionales con los estilos de aprendizaje de los estudiantes, de forma que los primeros favorezcan y mejoren todos los estilos de los segundos.

Para los autores Choque-Larrauri \& Chirinos-Cáceres (2009), las habilidades para la vida son habilidades personales, interpersonales, cognitivas y físicas que permiten a las personas controlar y dirigir sus vidas, desarrollando la capacidad para vivir con su entorno y lograr que éste cambie. Como ejemplos de habilidades para la vida individuales se pueden citar la toma de decisiones y solución de problemas, el pensamiento creativo y crítico, el conocimiento de sí mismo y la empatía, las habilidades de comunicación y de relación interpersonal y la capacidad para hacer frente a las emociones y manejar el estrés.

Las habilidades para la vida son un enfoque que desarrolla destrezas para permitir que los adolescentes adquieran las aptitudes necesarias para el desarrollo humano y para enfrentar en forma efectiva los retos de la vida diaria. Identifican tres clases de habilidades: 1) habilidades sociales o interpersonales (incluyendo comunicación, habilidades de rechazo, asertividad, agresividad y empatía), 2) habilidades cognitivas (incluyendo la toma de decisiones, el pensamiento crítico y la auto evaluación), y 3) habilidades para manejar emociones, incluyendo el estrés y aumento interno de un centro de control (Choque-Larrauri \& Chirinos-Cáceres, 2009). 
Adolescentes e internet

En investigación de Giménez-Gualdo, Maquilón-Sánchez \& Arnaiz (2014) los resultados muestran un alto índice de accesibilidad al móvil y al ordenador por parte de los adolescentes, situándose el consumo entre una y cuatro horas diarias. El principal uso de ambos medios es la comunicación y el entretenimiento, confirmándose además la influencia del sexo y el nivel educativo. Al analizar la prevalencia del cyberbullying aparecen diferencias estadísticamente significativas según sexo y rendimiento académico, lo cual confirma que el uso malintencionado de las tecnologías requiere de labor preventiva y de una intervención directa por parte de las familias y los centros educativos.

Por su parte, en el estudio en adolescentes de Delgado, Rojas, Salazar, Sancho \& Villalobos (2014), en el que se indagó sobre el autocuidado de este sector de la población ante el uso de redes sociales virtuales; se obtiene que la capacidad para el autocuidado se debe promover desde edades muy tempranas, siendo partícipes la familia, la comunidad, la sociedad, así como profesionales en orientación, quienes tienen como principio fundamental trabajar desde la prevención.

Además, se evidencia que las redes sociales son medios muy beneficiosos para la población adolescente en cuanto a sus relaciones interpersonales, pero si no se hace un uso correcto de las mismas, los daños en la persona pueden ser muchos, principalmente en la autoestima del adolescente (Delgado et al, 2014).

Hall (2010), evidencia en su estudio cuyo objetivo principal fue evaluar el grado de interacción social en las decisiones de educación de los adolescentes en Costa Rica; un alto grado de interacción entre los adolescentes al decidir sobre su nivel de escolaridad. Los resultados son robustos a la presencia de una serie de variables de control que buscan reducir el efecto de posibles variables no observables que pueden influenciar este tipo de decisión. En particular, incluye variables de control a nivel de la familia, del colegio y algunos efectos geográficos.

\section{Adolescentes y vocación}

En las decisiones relacionadas con su educación, los adolescentes no solamente toman en consideración sus características personales o las de sus familias, sino también las decisiones de educación de otros adolescentes cercanos a ellos. La forma en que estas interacciones sociales se llevan cabo puede ser muy diversas. Por ejemplo, éstas pueden ser directas, en las cuales los adolescentes se ayudan entre ellos a aprender en la escuela; o podrían ser indirectas en las cuales 
los adolescentes responden a las normas y estigmas desarrollados por los miembros del grupo (Hall, 2010).

Para González (s.f), la psicología moderna ha avanzado mucho en el estudio de las necesidades básicas del ser humano y su efecto decisivo de la conducta. Tales necesidades abarcan las físicas o biológicas, como el alimento, el abrigo, el descanso, hasta la necesidad de los valores intangibles, como la seguridad económica y social, la armonía con la realidad.

Gracia (2015), revela resultados del estudio en Alicante en los que se evidencian relaciones significativas entre el factor Apertura y el interés humanístico y científico-tecnológico, entre Extraversión y las áreas deportivas y de seguridad y entre Inestabilidad Emocional y los ámbitos psicopedagógico y sociojurídico. También se encontró que las áreas vocacionales preferidas por las mujeres son la psicopedagógica y la biosanitaria y que los hombres se decantan por la deportiva y las fuerzas de seguridad. La Inestabilidad Emocional es el único factor de personalidad en el que se hallaron diferencias significativas entre hombres y mujeres, siendo ellas quienes presentan una mayor inestabilidad.

Es importante que los adolescentes puedan trazar de forma consciente su recorrido vital para afianzar paulatinamente sus aspiraciones académicas y laborales, de las que debe ser conocedor el profesorado y progenitores. En estudio reciente en España, se detectan diferencias por género en cuanto a la inclinación revelada por el alumnado hacia asignaturas de ciencias experimentales o de lenguas. Además, los y las adolescentes se decantan principalmente por las profesiones relacionadas con el sector servicios (Ricoy \& Sánchez-Martínez, 2016).

Vásquez, \& Manassero (2008), indican que algunas medidas para mejorar la orientación académica y profesional, en relación con la ciencia y la tecnología en la secundaria podrían ser las siguientes: Fortalecer la coordinación entre los departamentos de ciencias y los de orientación, para promover redes, actividades e información. Priorizar actividades que estimulen el interés hacia la ciencia y la tecnología. Explicitar las relaciones entre los temas de ciencias y los problemas sociales, medioambientales, domésticos y laborales. Promover contactos y relaciones con empresas. Ofrecer a los estudiantes experiencias laborales en ciencias e ingenierías. Trabajar con las familias para ayudarles a comprender la importancia de las elecciones escolares.

Por último, sobre la proyección de adolescentes de secundaria para cursar estudios superiores, la investigación de Morales \& Pérez (2003) en Tenerife, destaca que en el caso de las mujeres solo el $51 \%$ de las estudiantes de centros públicos aspira realizar estudios universitarios, 
frente a un $82 \%$ obtenido para el caso de las estudiantes de centros educativos privados. En el caso de los hombres los datos son más alarmantes en el sector público, ya que solo el 34\% de los estudiantes de colegios públicos aspiran continuar con estudios universitarios, frente a un $82 \%$ de los estudiantes de colegios privados.

\section{Metodología}

La investigación se realizó recopilando información de estudiantes adolescentes de secundaria, por medio de un instrumento de consulta de creación propia del autor, con el fin de extraer información relevante en temas actuales de interés social, en diversas líneas de enfoque, con el fin de analizar y apoyar en la orientación del complejo mundo del adolescente, para la oportuna toma decisiones.

A grandes rasgos, las preguntas abarcaron generalidades demográficas, consultas de afinidad por ciertas materias, preferencias en el proceso enseñanza-aprendizaje, interrogantes sobre uso de dispositivos y aplicaciones electrónicas, decisiones y afinidad por cursar estudios superiores, centros de estudios superiores preferidos y motivo de elección del mismo, entre otras.

Se contemplaron estudiantes de secundaria, de tres colegios del Ministerio de Educación Pública de la zona de Alajuela, Costa Rica; específicamente el Liceo de Atenas, el Liceo de Santa Gertrudis de Grecia y el Liceo del Pacto del Jocote. En el total de la muestra se consideró abarcar aleatoriamente hombres y mujeres de todos los niveles de secundaria, con el fin de lograr representatividad en los resultados.

La información se recopiló de manera anónima y voluntaria, guardando por completo la integridad e identidad de los adolescentes participantes. Luego se tabuló, graficó y analizó en Excel para reflejar la realidad de los adolescentes de la zona considerada en el estudio, para los temas implicados.

Resultados y discusión

Una vez tabulada la información en Excel, se procedió con el análisis y discusión respectiva. Como se observa en la Figura 1A, se incluyeron un total de 102 estudiantes, de los cuales 42 son hombres y 60 mujeres. Asimismo se evidencia la distribución de hombres y mujeres según nivel de secundaria que cursan, siendo en todos los casos, mayor la cantidad de mujeres por grupo encuestado según nivel. 
A

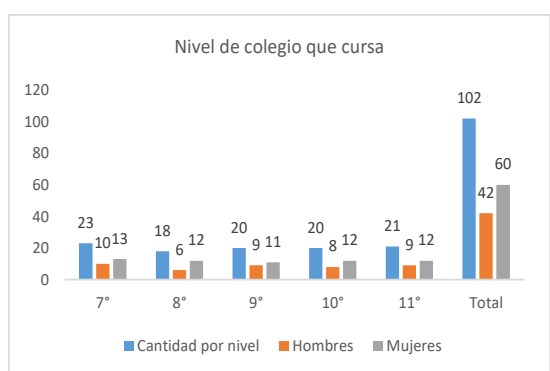

B

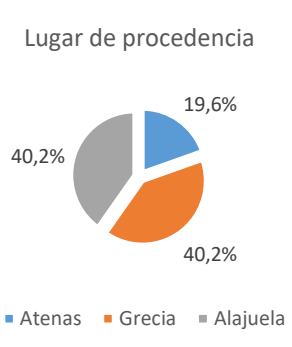

$\mathrm{C}$ Edad en años cumplidos

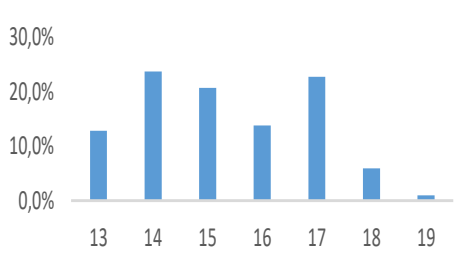

Figura 1. Distribución de estudiantes encuestados por nivel y sexo (A), lugar de procedencia (B) y edad (C).

Según el lugar de procedencia, se observa en la Figura 1B, que un 40\% de los adolescentes son de la zona de Grecia, un $20 \%$ de la zona de Atenas y otro $40 \%$ provenientes de Alajuela. A su vez, la Figura 1C refleja la distribución de los encuestados por edad en años cumplidos, concentrándose la mayoría entre 14 y 17 años de edad.

Ante la consulta de cuál asignatura consideran más difícil en su nivel respectivo, la Figura $2 \mathrm{~A}$ ilustra el comportamiento en $7^{\circ}, 8^{\circ}$ y $9^{\circ}$ año, donde en sétimo la dificultad la perciben muy similar entre Matemática y Estudios Sociales, mientras que en octavo y noveno, al igual que en $10^{\circ}$ y $11^{\circ}$ (Figura 2B) sobresale la percepción de dificultad por la Matemática en los adolescentes. El $64 \%$ de los adolescentes incluidos en el estudio consideran la Matemática como la materia más difícil.

A $\quad$ B

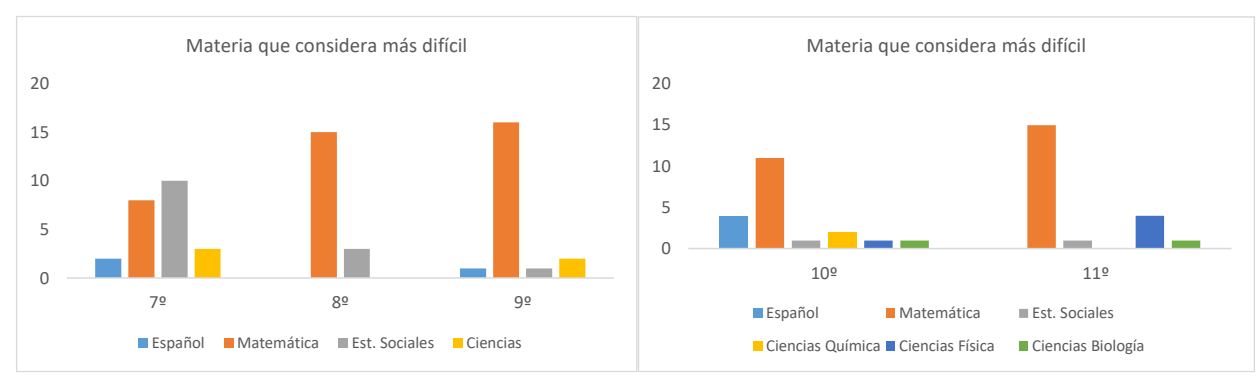

$\mathrm{C}$

$\mathrm{D}$ 


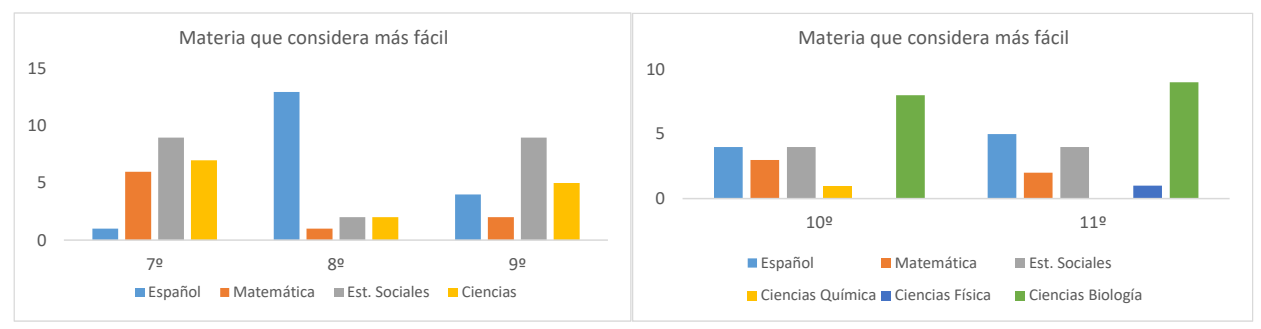

Figura 2. Asignatura considerada más difícil por los adolescentes encuestados en $7^{\circ}, 8^{\circ}$ y $9^{\circ}(\mathrm{A}), \mathrm{y}$ en $10^{\circ}$ y $11^{\circ}(\mathrm{B})$ año de secundaria. Materia considerada más fácil en $7^{\circ}, 8^{\circ}$ y $9^{\circ}(\mathrm{C})$ y en $10^{\circ}$ y $11^{\circ}$ (D).

En contraposición, lo obtenido para la consulta de cuál materia consideran más fácil, se observa en la Figura 2C que para los adolescentes de sétimo y noveno año es Estudios Sociales y para los de octavo es Español. En décimo y undécimo año sobresale Biología como el área que perciben más fácil los adolescentes (Figura 2D). En términos generales las Ciencias, Español y Estudios Sociales son las materias en las que más coinciden como fácil los estudiantes, con un 32, 28 y $27 \%$ de los participantes del estudio respectivamente.

En relación con las consultas anteriores, se hizo la consulta de cuál materia les gusta más, con el fin de relacionar con la materia que consideraron más fácil. La Figura 3A evidencia que de sétimo a noveno año la materia más gustada es Ciencias, y en décimo y undécimo año se refuerza esa inclinación con la afinidad en el gusto por Biología. En general un 47\% de los adolescentes eligieron el área de las Ciencias como la materia que más les gusta, seguido de Estudios Sociales con un $25 \%$. Esto coincide con las materias que los adolescentes perciben más fáciles.

A

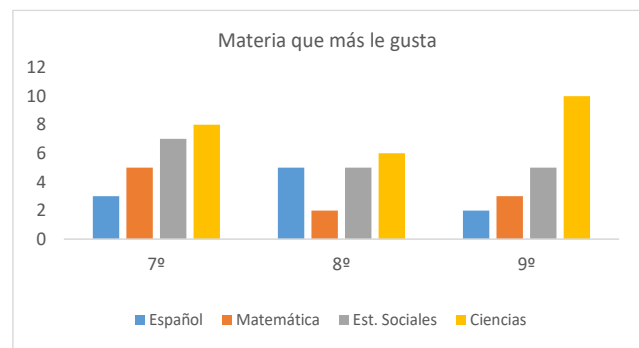

$\mathrm{C}$
B

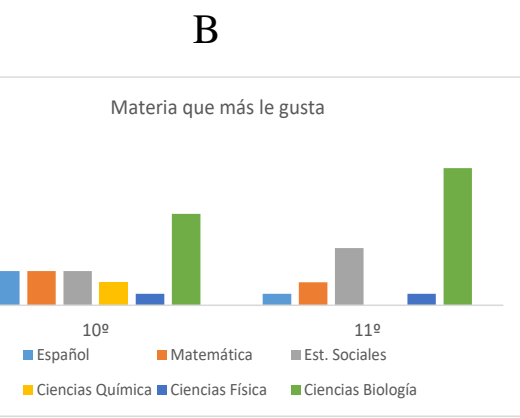

$\mathrm{D}$ 


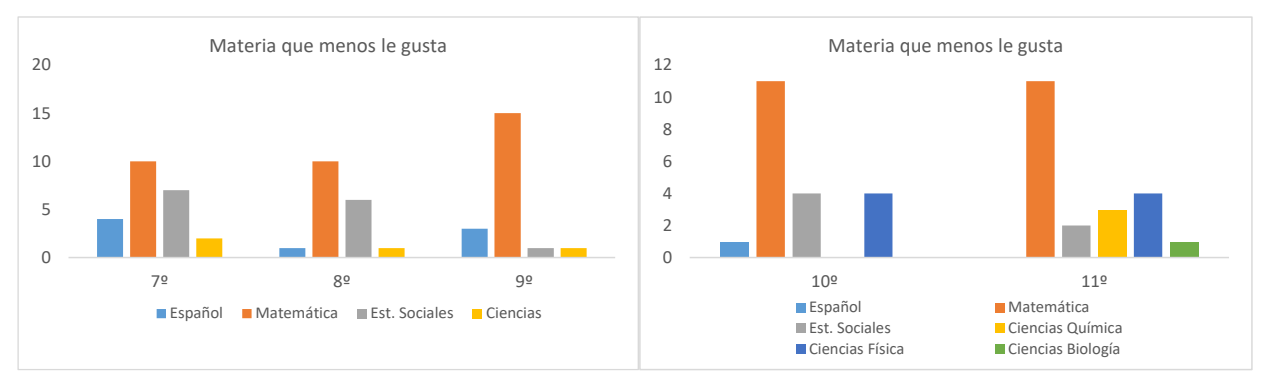

Figura 3. Materia que más les gusta a los adolescentes encuestados en $7^{\circ}, 8^{\circ}$ y $9^{\circ}(\mathrm{A})$, y en $10^{\circ}$ y

$11^{\circ}(\mathrm{B})$ año de secundaria. Materia que menos les gusta en $7^{\circ}, 8^{\circ}$ y $9^{\circ}(\mathrm{C})$ y en $10^{\circ}$ y $11^{\circ}(\mathrm{D})$.

Por su parte las Figuras 3C y 3D destacan la asignatura Matemática como la que menos gusta en los adolescentes estudiantes de los diferentes niveles de secundaria. Un 56\% del total de encuestados indicaron que la Matemática es la materia que menos les gusta, seguido de Estudios Sociales con un $20 \%$.

Ante la consulta de cuál área de las Ciencias seleccionaría para el examen final de bachillerato, la Figura 4 destaca que un $47 \%$ aún no saben (principalmente los de $7^{\circ}$ y $8^{\circ}$ ) y un $44 \%$ se inclinarían por Biología. En los años clave de décimo y undécimo se tiene que solo 5 de 20 y 1 de 21 respectivamente, son los que no saben que presentar al momento de responder el instrumento de consulta. Destaca en estos dos últimos niveles que solo dos presentarían Química, uno presentaría Física y 32 lo harían por Biología (78\% de los estudiantes de $10^{\circ}$ y $11^{\circ}$ ). Para ChoqueLarrauri \& Chirinos-Cáceres, 2009, en relación a la habilidad de toma de decisiones en adolescentes se menciona que esta habilidad es un proceso complicado, puesto que requiere de un análisis afectivo, emocional y cognitivo. La toma de decisiones implica elegir la mejor opción para solucionar una dificultad y tener una facultad para comprender entre varias opciones cuál es la mejor. 


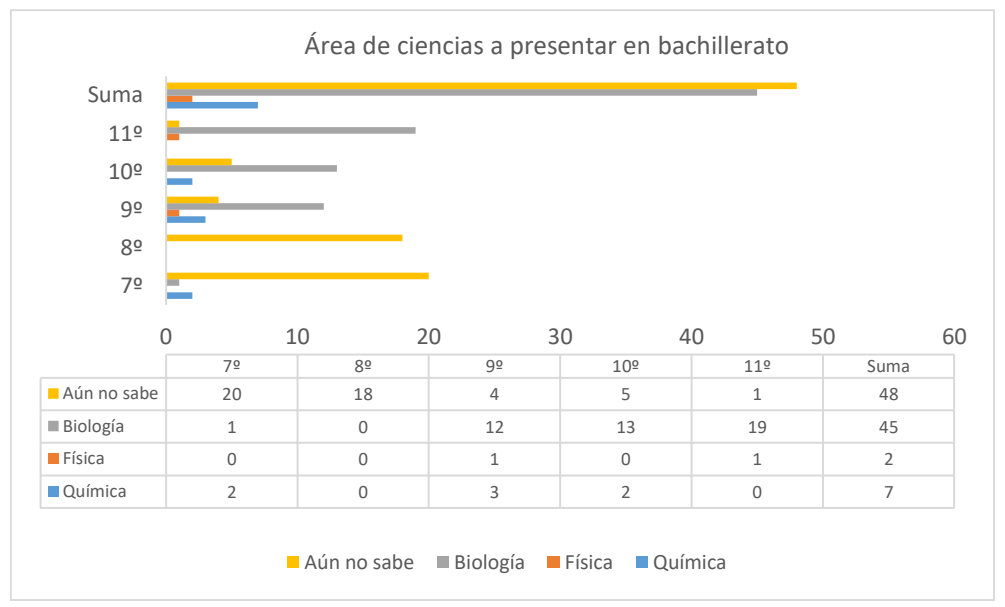

Figura 4. Área de las ciencias preferida para el examen final de bachillerato en estudiantes de secundaria.

En otro tema de suma relevancia, se le consultó a los adolescentes sobre la modalidad de enseñanza preferida en su clase, con el fin de hacer la clase lo más enriquecedora y satisfactoria según su percepción. La figura 5 revela los resultados, entre los cuales destaca: el 58\% de los adolescentes que prefieren la asignación de trabajos en grupo y exposición de los estudiantes como la opción más adecuada.

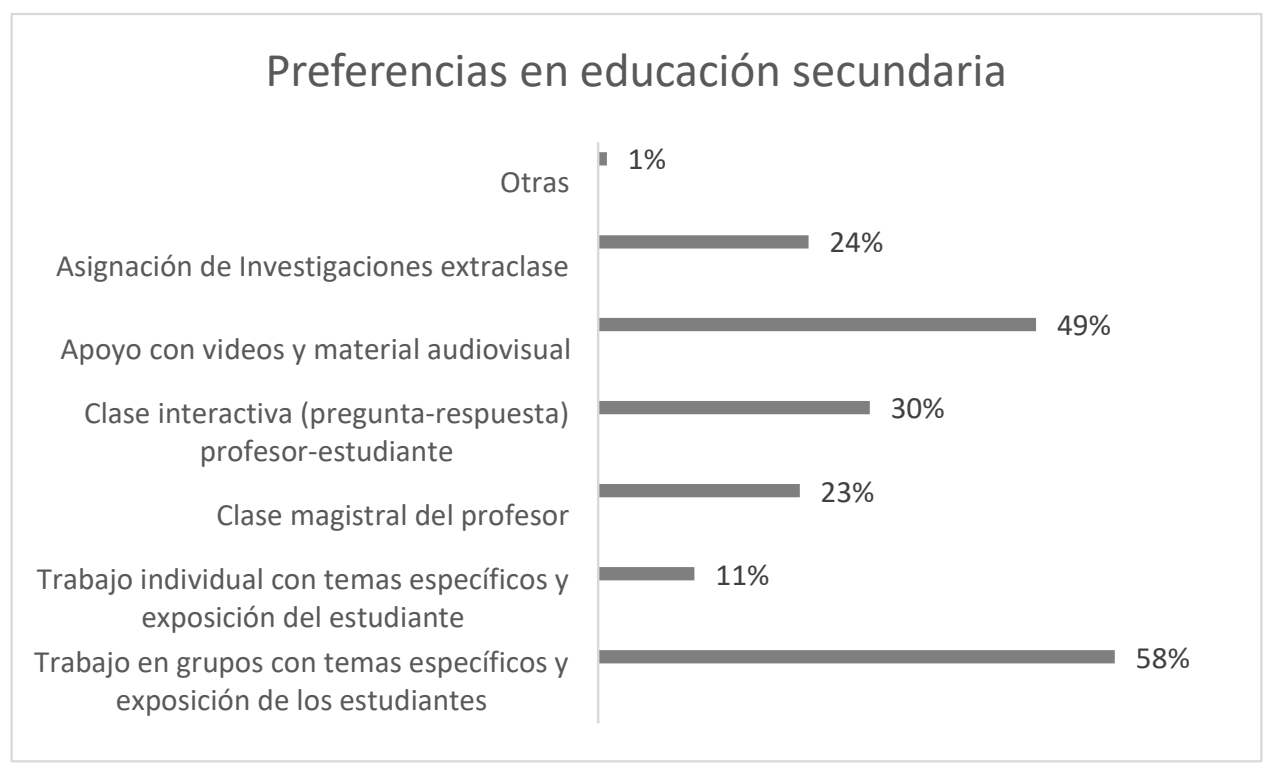

Figura 5. Preferencias educacionales de trabajo para los estudiantes de secundaria. 
En segundo lugar de preferencia el $49 \%$ de los estudiantes escogieron el apoyo con videos y material audiovisual para la realización de la clase. El trabajo individual con exposición del estudiante fue percibido como la alternativa menos apropiada para el proceso enseñanzaaprendizaje con solo un 11\%, junto con las opciones adicionales propuestas por los mismos estudiantes lo cual se indicó con la leyenda “Otras” (1\%). Bibiano, Vázquez \& Ramírez (2015) mencionan que la lectura comprensiva, promueve en el adolescente lector la sistematicidad y la coherencia, más que el lenguaje oral que literalmente puede variar mucho gracias al manejo del contexto inmediato.

En lo que respecta al uso de dispositivos electrónicos básicos, así como tecnologías de la información de uso frecuente en la sociedad actual, la Figura 6 ilustra que el 100\% de los adolescentes encuestados cuenta con teléfono celular, el $95 \%$ de ellos cuenta con internet en su dispositivo móvil; el $80 \%$ tiene computadora en su casa, y el 98\% indicaron que utilizan el internet para labores estudiantiles. El 73\% de los estudiantes indicó utilizar correo electrónico y el total de los adolescentes que usan internet indicaron utilizar Google como el buscador de internet preferido. Estos resultados reflejan el uso masivo de dispositivos electrónicos y el uso trascendente del internet para tareas educativas en los estudiantes de secundaria en la zona de Alajuela. Se debe resaltar, que el internet ocupa una gran parte del tiempo de los adolescentes, especialmente como medio de comunicación, con WhatsApp y las redes sociales como las aplicaciones que utilizan con más frecuencia (Reolid-Martínez, Flores-Copete, López-García, Alcantud-Lozano, Ayuso-Raya, \& Escobar-Rabadán, 2016).

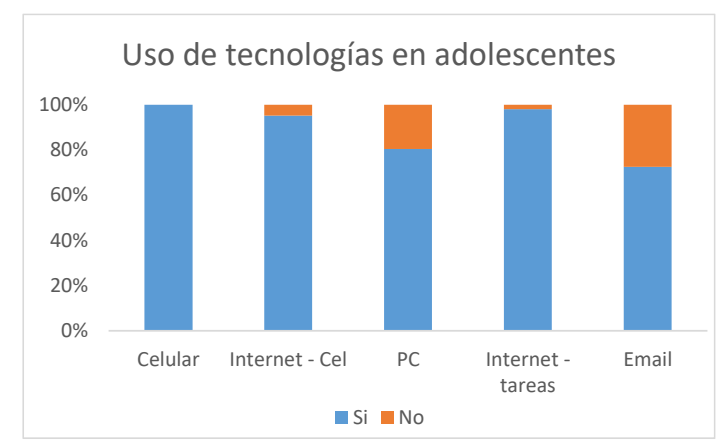

Figura 6. Uso de tecnologías básicas en adolescentes de secundaria.

Por su parte la Figura 7A, ilustra el uso de las redes sociales por los adolescentes incluidos en la investigación, según el nivel de secundaria que cursan. Sobresale el $89 \%$ en el octavo nivel 
con el menor porcentaje de uso, versus el 100\% que utilizan al menos una de ellas, obtenido para los estudiantes de undécimo nivel de secundaria. A su vez, sobre la preferencia de las redes sociales en los adolescentes, la Figura 7B destaca el uso de Facebook con un 94\% que la utilizan y Whatsapp con un 54\%. Estas como las más utilizadas por los alumnos consultados, que indicaron hacer uso de al menos una de ellas. Además, un 52\% de los estudiantes de colegio hicieron referencia al uso de otras redes sociales de manera paralela a, al menos una de las dos antes señaladas. Los resultados evidencian la predilección por las redes sociales, donde solo un 6\% del total de participantes del estudio no utilizan ninguna red social, lo cual podemos vincular de manera estrecha con el $5 \%$ de adolescentes que no cuentan con internet en su celular.

A

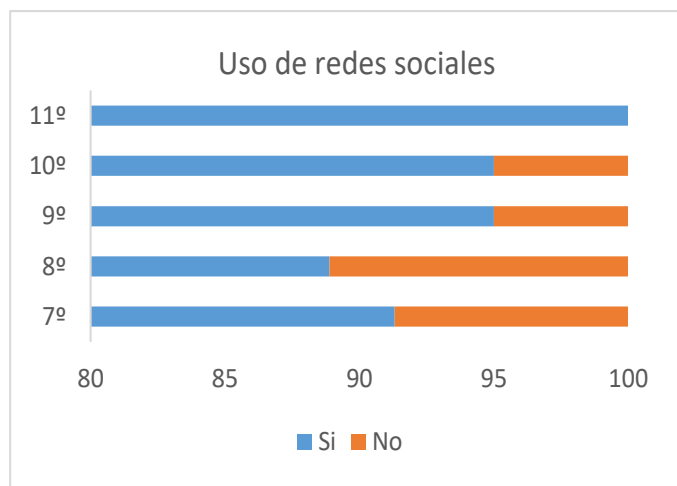

B

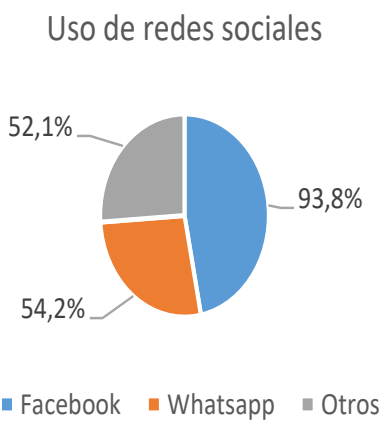

Figura 7. Uso de redes sociales en adolescentes de secundaria.

En temas de elección vocacional futura, según Ponti, Sanchez, Luján \& Cervetto (2012), las intervenciones de la orientación vocacional en adolescentes, apuntan a promover en los jóvenes la construcción de la identidad vocacional-ocupacional, colaborando con el bienestar emocional. Ayudar a encontrar "un saber y un hacer" que les permita insertarse en la comunidad de un modo productivo y autónomo, favorece el sentido de pertenencia a dicha comunidad y, al mismo tiempo, posibilita a la persona el desarrollo de su sí mismo.

Se le consultó a los estudiantes de secundaria sobre su intención de cursar estudios superiores, a lo cual el 100\% indicaron que sí lo harán. Adicionalmente, el 81\% de los estudiantes ya tienen claro qué les gustaría estudiar; la Figura 8A demuestra la tendencia por nivel de secundaria ante la orientación sobre la decisión de la carrera que desean estudiar los consultados; como se observa no hay mayor variante de un nivel a otro destacando el $22 \%$ de estudiantes que no saben aún qué estudiar de los niveles sétimo y octavo, versus el 14\% del mismo aspecto en 
undécimo nivel, lo cual sí puede considerarse de cuidado por ser quienes estarían en la antesala de elegir carrera para el siguiente año iniciar sus estudios superiores. Esto se debe destacar por la necesidad de orientar con mayor esfuerzo y dedicación a este sector de secundaria que le urge aclarar su percepción y tomar una decisión informada en temas vocacionales. Por su parte la Figura 8B evidencia que el principal motivo de elección de carrera fue por la afinidad con la profesión con un $30 \%$, seguido del buen salario que ostenta el profesional con un $26 \%$ según la percepción de los adolescentes consultados.
A
B

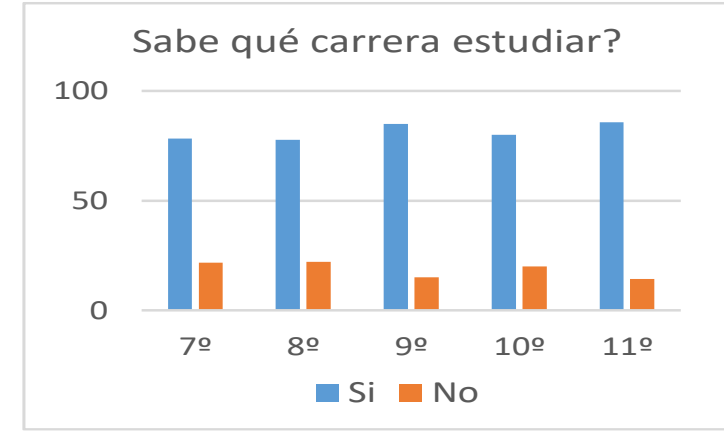

$\mathrm{C}$

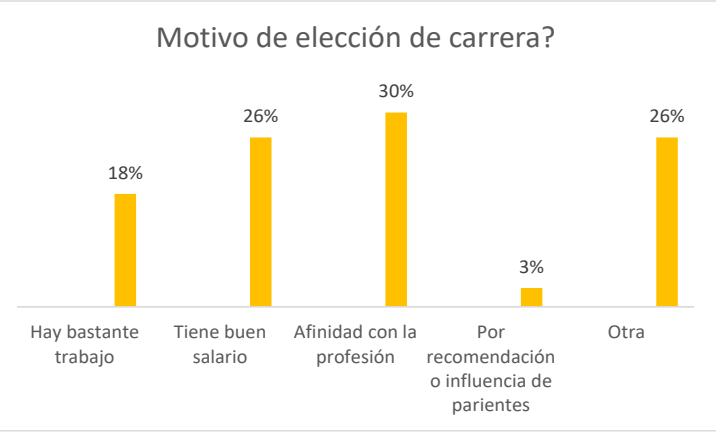

D

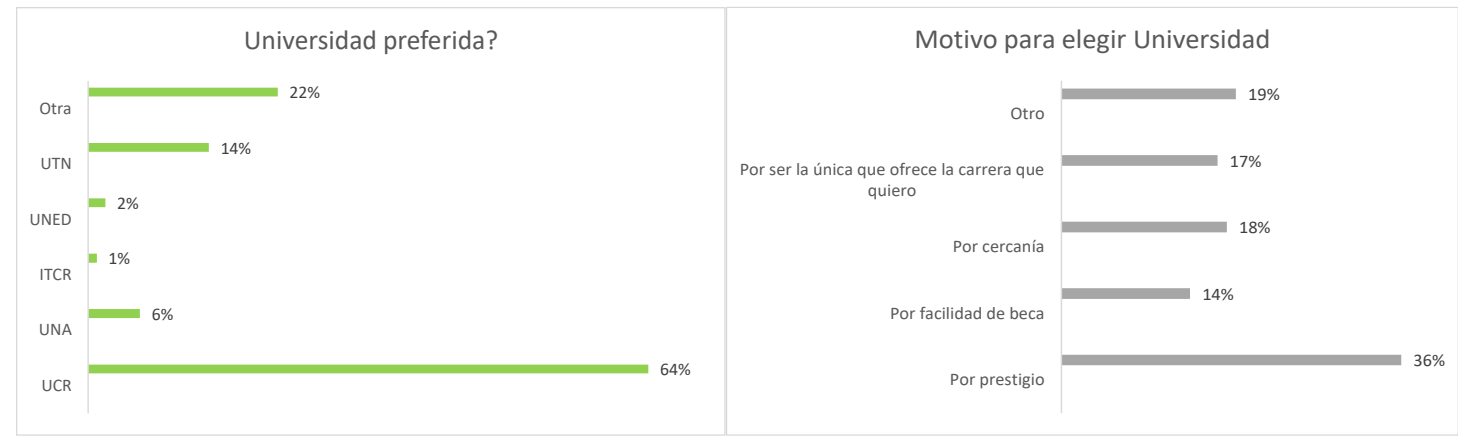

E

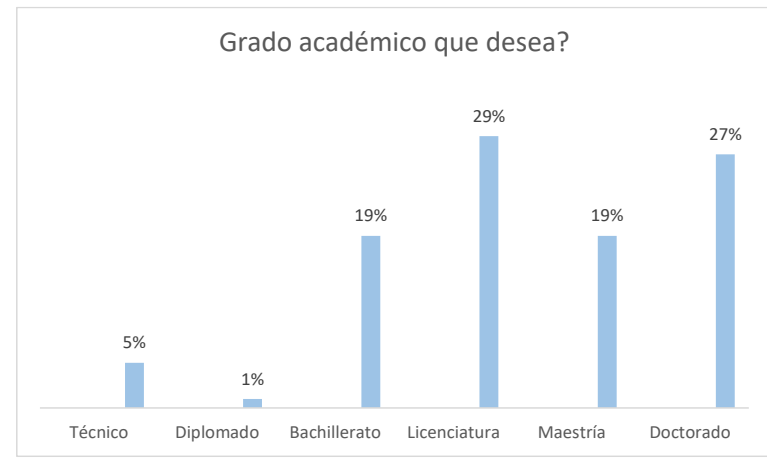

Figura 8. Orientación vocacional en estudiantes de secundaria. 
Asimismo como se ve en la Figura 8C la universidad preferida para los adolescentes es la Universidad de Costa Rica (UCR) con un 64\% de los encuestados que la eligieron, a lo cual indicaron que el prestigio es la principal razón de elección de esta universidad, con un 36\% del total de estudiantes participantes que se inclinaron por esta opción (ver Figura 8D).

Por último, se les consultó sobre el grado académico que desean obtener en sus estudios superiores y en los resultados sobresale la licenciatura, seguido del doctorado como los niveles académicos preferidos por los estudiantes, con 29 y 27\% (Figura 8E) del total de participantes en la investigación.

\section{Conclusiones}

- El estudio revela la percepción de dificultad de los estudiantes, ante las matemáticas en los diferentes niveles de secundaria en los colegios considerados de la provincia de Alajuela, Costa Rica.

- Las materias Ciencias, Español y Estudios Sociales son las que perciben como más fácil; y el área de las Ciencias es la que más gusta entre los adolescentes de secundaria.

- El 58\% de los adolescentes prefieren la asignación de trabajos en grupo y exposición de los estudiantes, como la opción más adecuada para el proceso enseñanza-aprendizaje en el aula.

- El 78\% de los estudiantes que cursan décimo y undécimo nivel seleccionarían Biología como el área de las Ciencias para el examen de bachillerato.

- Estudios como este revelan la realidad puntual de las nuevas generaciones en temas educacionales en edad adolescente, lo que permite a la sociedad enfocar esfuerzos en atender tan importante sector de la población, que marcarán el destino de todo un país en el corto plazo.

- El total de los estudiantes de secundaria incluidos en el estudio, cuentan con teléfono celular (95\% con internet en el dispositivo), el 98\% de ellos indicó utilizar internet para tareas del colegio, el $80 \%$ tienen computadora en la casa y $73 \%$ utilizan correo electrónico.

- Los resultados evidencian la predilección por el uso de redes sociales, con tan solo un $6 \%$ del total de participantes del estudio que no usan ninguna red social, lo cual se puede vincular de manera estrecha con el $5 \%$ de adolescentes que no cuenta con internet en su celular. Facebook es la red social más utilizada, seguida de Whatsapp. 
- El $81 \%$ de los estudiantes ya saben qué elegirán como carrera universitaria, destacan el $22 \%$ de estudiantes de los niveles sétimo y octavo que no saben aún qué estudiar, y el 14\% del mismo aspecto en undécimo nivel.

- El principal motivo de elección de carrera sería por la afinidad con la profesión con un 30\%, seguido del buen salario que ostenta el profesional con un $26 \%$ según percepción de los estudiantes. La universidad preferida por los estudiantes de secundaria es la Universidad de Costa Rica, por el prestigio de esta; y el principal grado académico que persiguen es licenciatura y doctorado.

- De esta forma resaltamos la relevancia de los resultados evidenciados en este estudio, como insumo de primera mano para la sociedad, familia y sector educación, que pueden ser utilizados por las autoridades estatales con el fin de tomar decisiones en orientación y mejora, en lo que respecta al desempeño cotidiano del adolescente promedio de secundaria en Costa Rica, y velar por sus derechos, deberes y protección ante riesgos y vulnerabilidades.

\section{Referencias bibliográficas}

Aguilar, J. M. S., Lozoya, S. E. B., \& Guzmán, V. C. (2015). La incorporación de la era digital en la adquisición de aprendizajes por proyectos en la materia de FCE-Secundaria. Colegio Madrid.

Alcázar, L., Rendón, S., \& Wachtenheim, E. (2001). Trabajando y estudiando en América Latina rural: decisiones críticas de la adolescencia. Madrid.

Artagaveytia, L. \& Bonetti, J. (2006). Educación y participación adolescente - Palabras y juegos. Unicef Uruguay.

Bertolino, M. F. (2011). Las condiciones objetivas y subjetivas de los adolescentes y sus trayectorias educativas. XI Jornadas Argentinas de Estudios de Población. Asociación de Estudios de Población de la Argentina, Neuquén.

Bibiano, S.; Vázquez, E. \& Ramírez, J. (2015). La comprensión lectora en adolescentes de secundaria. Alternativas para nuevas prácticas educativas Libro 1. Estrategias para la transformación de la enseñanza de la lectura. Editorial Amapsi. México, 76. 
Choque-Larrauri, R., \& Chirinos-Cáceres, J. (2009). Eficacia del Programa de habilidades para la vida en adolescentes escolares de Huancavelica, Perú. Revista de Salud pública, 11(2), 169181.

Delgado, C., Rojas, E., Salazar, A., Sancho, A. \& Villalobos, P. (2014). El papel de la persona profesional en Orientación sobre el desarrollo de la capacidad de auto cuidado de adolescentes que utilizan las redes sociales virtuales. Memoria de Seminario de Graduación para optar por el grado de Licenciatura en Ciencias de la Educación con énfasis en Orientación. Universidad de Costa Rica.

Delors, J., Amagi, I., Carneiro, R., Chung, F., Geremek, B., Gorham, W., \& Stavenhagen, R. (1997). La educación encierra un tesoro: informe para la UNESCO de la Comisión Internacional sobre la Educación para el Siglo Veintiuno. Unesco.

Fraguela, R., Varela, L., \& Caballo, M.B. (2016). Percepción del profesorado de Enseñanza Secundaria Postobligatoria sobre el papel de la escuela y sus tiempos en el ocio del alumnado. Revista Interuniversitaria de Formación del Profesorado, 86 (30.2), 17-31.

García, J.D. (2008). Clases sociales e identidad personal: estudio comparativo de la construcción y formación de la identidad personal en adolescentes de dos clases sociales, mujeres y hombres, de 16 y 17 años, de San José. Tesis de Licenciatura en Psicología. Universidad de Costa Rica.

Giménez-Gualdo, A., Maquilón-Sánchez, J. \& Arnaiz, P. (2014). Acceso a las tecnologías, rendimiento académico y cyberbullying en escolares de secundaria. Revista iberoamericana de psicología y salud, 5(2), 119-133.

Gómez-Vela, M., Verdugo, M. Á., \& González-Gil, F. (2007). Calidad de vida y autoconcepto en adolescentes con necesidades educativas especiales y sin ellas. Infancia y Aprendizaje, 30(4), 523-536.

González, E. (s.f). Las Necesidades del Adolescente. Revista de la Universidad de Costa Rica. Número 9. 109-117.

Gracia, P. (2015). Personalidad y preferencias vocacionales en estudiantes de Secundaria. Trabajo Final de Grado en Psicología.

Gutiérrez, M., \& Piedra, N. (2008). VI Informe del Estado de los Derechos de la Niñez y la Adolescencia en Costa Rica. UCR-UNICEF. 
Hall, L. (2010). Efectos de la Interacción Social en las Decisiones sobre Educación de los Adolescentes en Costa Rica. Ciencias Económicas 28-No. 2,323-334.

Krichesky, M. (2005). Adolescentes e inclusión educativa: un derecho en cuestión. Noveduc Libros.

López, F., \& Angulo, R. (2006). La Reforma de la Educación Secundaria en México. Revista mexicana de investigación educativa, 11(31), 1427-1450.

Morales, M. O. G., \& Pérez, F. M. D. (2003). Valoración de las expectativas educativas y profesionales de los estudiantes de los centros de Educación Secundaria según su naturaleza pública o privada. In Hacienda pública y convergencia europea: X Encuentro de Economía Pública, Santa Cruz de Tenerife (p. 76). Universidad de La Laguna.

Pacheco, V. (2001). El desarrollo del talento sobresaliente en los estudiantes adolescentes. Revista Educación, Vol. 25, Núm. 1, 123-135.

Ponti, L., Sanchez, M., Luján, S., \& Cervetto, J. (2012). Aportes de los talleres de orientación vocacional en la elección vocacional desde la perspectiva de los adolescentes. IV Congreso Internacional de Investigación y Práctica Profesional en Psicología. Facultad de PsicologíaUniversidad de Buenos Aires.

Quintanal-Pérez, F. Q. (2012). Relación entre estilos de aprendizaje y rendimiento escolar en física y química de secundaria. Vivat Academia, (117E), 1143-1153.

Reolid-Martínez, R. E., Flores-Copete, M., López-García, M., Alcantud-Lozano, P., Ayuso-Raya, M. C., \& Escobar-Rabadán, F. (2016). Frecuencia y características de uso de Internet por adolescentes españoles: Un estudio transversal. Archivos argentinos de pediatría, 114(1), 6-13.

Ricoy, M. C., \& Sánchez-Martínez, C. (2016). Preferencias académicas y laborales en la adolescencia: Una perspectiva de género. Estudios pedagógicos (Valdivia), 42(2), 299-313.

Unicef. (2011). Estado mundial de la infancia 2011-Resumen Ejecutivo: La adolescencia una época de Oportunidades. UNICEF.

Vázquez, Á., \& Manassero, M. A. (2008). El declive de las actitudes hacia la ciencia de los estudiantes: un indicador inquietante para la educación científica. Revista Eureka, 5(3), 274-292.

Vásquez, A., \& Manassero, M. A. (2008). La elección de asignaturas de ciencias: análisis de los factores determinantes. Revista Española de Pedagogía, Vol. 66, No. 241, 541-558. 
InterSedes, $\mathbf{N}^{\circ}$ 42. Vol XX (2019). ISSN 2215-2458

Villaseñor, L. M. (2006). Lectura y educación: los hábitos lectores y su repercusión académica en Educación Secundaria Obligatoria. Ocnos: Revista de estudios sobre lectura, (2), 105-122. 